\title{
Host genetic diversity influences the severity of Pseudomonas aeruginosa pneumonia in the Collaborative Cross mice
}

\author{
Nicola Ivan Lorè ${ }^{{ }^{*}}$, Fuad A Iraqi $i^{2 \dagger}$ and Alessandra Bragonzi ${ }^{1^{* \dagger}}$
}

\begin{abstract}
Background: Pseudomonas aeruginosa is one of the top three causes of opportunistic infections in humans. Patients with a compromised immune system, due to immunosuppressive therapies or underlying diseases such as cancer, AIDS or the hereditary disease cystic fibrosis, are at risk of developing $P$. aeruginosa infection. However, clinical evidence indicates extremely variable outcomes of $P$. aeruginosa infections in individuals at risk, suggesting that host multi-complex genetic traits may influence the severity of this opportunistic infection. Here, we have used an innovative experimental model to dissect whether host genetic background, such as those found in the outbred population, could influence the risk of morbidity and mortality to $P$. aeruginosa pneumonia.
\end{abstract}

Results: A highly genetically-diverse mouse resource population, Collaborative Cross (CC) mice, was infected with a clinical strain of $P$. aeruginosa and subsequently monitored for mortality, mean survival time, and morbidity, change in body weight for seven days post infection. Disease phenotypes ranged from complete resistance and recovery of body weight to lethal disease. Initial variables, including body weight, age and gender, have limited influence on $P$. aeruginosa outcome, emphasizing the role of host genetic background in defining the risk of morbidity and mortality. When broad-sense heritability of phenotypic traits was evaluated, it confirmed the influence of genetic profile rather than environmental factors among the CC lines during $P$. aeruginosa infection.

Conclusion: This innovative model system can potentially reproduce the variables responses of disease severity observed in humans during P. aeruginosa pneumonia. Our results demonstrated that a widely-marked differential response to $P$. aeruginosa airway infection in term of morbidity and mortality, is mainly affected by host genetic factors, as multiple genetic loci or polymorphic variations.

Keywords: P. aeruginosa, Acute infection, CC mice, Disease phenotype, Airway infection, Animal model, Forward genetics

\section{Background}

Genotype is an important determinant factor in human host susceptibility to major diseases, including infections [1]. There is increasing evidence that some inter-population and inter-individual differences in the attack rate and prognosis of specific infectious organisms are due to inherited genetic variants and, for the most part, to multicomplex genetic traits (polygenetic traits) [2]. These findings are changing our view of infections as we can now assume that

\footnotetext{
*Correspondence: lore.nicolaivan@hsr.it; bragonzi.alessandra@hsr.it

${ }^{\dagger}$ Equal contributors

${ }^{1}$ Infection and Cystic Fibrosis Unit, Division of Immunology, Transplantation and Infectious Diseases, IRCCS - San Raffaele Scientific Institute, Milan, Italy Full list of author information is available at the end of the article
}

pathogens are not the sole determinants of the corresponding infectious diseases. Host response is influenced by the complex combinations and variations of genes, and this affects the outcome of the infectious disease. An efficient experimental method to dissect complex genetic traits still needs to be established. Recently, a new community resource - Collaborative Cross (CC) mice - has been implemented as a common platform for mammalian complex genetic traits in an attempt to overcome the limitation of existing resources [3, 4]. The $\mathrm{CC}$ is a murine reference population with high genetic diversity, constructed using a randomized breeding design that systematically outcrosses eight founder strains, followed by inbreeding to obtain new recombinant inbred strains. Five 
of the eight founders are common laboratory strains (A/J, C57BL/6 J, 129S1/SvImJ, NOD/LtJ, NZO/HiLtJ), and three are wild-derived strains (CAST/EiJ, PWK/PhJ, and WSB/ EiJ) [5]. The $\mathrm{CC}$ has been genotyped and showed more recombination and genetic variation compared to the other reference panels [6]. The genomes of the CC founder strains (http://www.sanger.ac.uk/resources/ mouse/genomes/) and CC lines strains (http://csbio. unc.edu/CCstatus/index.py) (http://mus.well.ox.ac.uk/ $\mathrm{CC} /$ ) have been genotyped or sequenced, so it is possible to reconstruct the haplotypes of each CC line as a fixed mosaic of the founder chromosomes $[6,7]$. Hallmarks of CC lines include high mapping resolution and sample sizes that are sufficient to drive phenotypic diversity in almost any trait of interest.

This paper reports the use of CC mice as an innovative forward genetic approach to determine whether and to what extent host genetic background, influences the variability of $P$. aeruginosa acute respiratory infection. $P$. aeruginosa retains a prominent position as a major worldwide cause of morbidity and mortality in a wide range of patients including those with a compromised immune system from immunosuppressive therapies or underlying diseases such as cancer, AIDS or the hereditary disease, cystic fibrosis (CF) [8-10]. Clinical evidence indicates that the outcome of $P$. aeruginosa infections may be extremely variable among individuals at risk, suggesting that host multicomplex genetic traits may influence the severity of this opportunistic infection. Genetic mapping in the human population has been performed, but partially achieved significance in the genome-wide association study [11]. Studies from CF twins and sibs have suggested that susceptibility to chronic infection with $P$. aeruginosa is affected by genetic inheritance determinants, whereas initial infection is minimally influenced by genetic modifiers [12]. However, the size of the cohorts, the strong but often unknown environmental influences, poor diagnosis and lack of repeatability are bottlenecks in human studies that highlight the need for novel resources [13-15]. Mouse inbred strains are the starting point from which to explore causal genotype-phenotype relationships and identify gene mapping however they do not offer a strong tool to identify and study polymorphic variations, that are hallmarks of the human population $[16,17]$. The number of classical inbred strains is relatively small. This limits genetic and phenotypic diversity and the value of Quantitative Traits Loci (QTL) detection. Thus, innovative experimental animal models are absolutely essential to complement the human studies [18]. Using CC mice, it is possible to determine genotype variation associated to disease phenotypic traits (e.g., using merge analysis) [6, 7]. Power and resolution mapping of $\mathrm{CC}$ mice was recently completed for host susceptibility to Aspergillus fumigatus [7], influenza A [19, 20], Klebsiella pneumoniae [21] and reported for the first time in this paper as proof-ofconcept for new studies to map QTL associated with the opportunistic bacteria $P$. aeruginosa.

\section{Results}

\section{Host genetic background influences the severity of} $P$. aeruginosa pneumonia

CC lines, infected with $P$. aeruginosa, showed a wide-range of Survival time (ST) ranging from complete resistance (100\% survival after seven days post-infection) to lethal disease (100\% death after 1.5 days), while A/J mice showed an intermediate phenotype (30\% of mortality rate after 7 days post-infection) (Fig. 1a, Additional files 1 and 2). Similarly, CC lines had a wide variation in body weight (BW) response to $P$. aeruginosa infection: ranging from a $23 \%$ decrease in BW after three days to those showing an almost total recovery of change in BW after five days (Fig. 1b). A/J mice lost $16 \%$ of their change in BW after three days but they did not recover completely after seven days. At day 7 post-infection, bacterial cells were not recovered in the organs (blood, liver and lung) of surviving mice (data not shown), indicating that bacterial clearance is independent from differences in morbidity as assessed by recovery of body weight. These data confirm that different traits of the CC mice resource population express a wide response to $P$. aeruginosa infection, suggesting the key role of genetic variance on the severity of the clinical outcome.

\section{Segregation analysis of disease phenotypic traits}

Phenotypic traits for segregation analysis of mean survival time (MST) and change in body weight at day one (CBW1) after $P$. aeruginosa airway infection were selected based on previous studies with commercial inbred strains [17, 22]. Thus, segregation analysis of the phenotypic trait distributions highlights the different responses among CC lines. Figure 2 shows MST and CBW1 of the CC lines, arranged by increasing order of magnitude. Based on the statistical significance using Bonferroni's Multiple Comparison Tests (reported in Additional file 3), CC lines were arranged by a similar variation degree of recorded traits in three groups (Fig. 2). Thus, in the high tail of MST, four CC lines (IL2156, IL521, IL2689, IL3438) were ranked with the highest MST value (MST: 5.3/7) while in the low tail, five CC lines (IL711, IL1061, IL188, IL2126, IL1912) showed the lowest MST values (MST: 1.41/1.48) (Fig. 2a). An intermediate group of eight murine lines (IL4052, IL611, IL72, IL111, IL3912, IL4457, IL4141, IL519) showed a medium MST value (MST: 1.6/4.5). Statistical analysis of CBW1 highlights a two-fold difference between the highest (IL4141, IL519, IL188; CBW1: $-7.22 \% /-9.33 \%$ ) and the lowest CC line groups (IL72, IL521, IL4457; CBW1: $-3.16 \% /-4.34 \%$ ) (Fig. 2b). An intermediate group of eleven murine lines (IL4052, IL2689, IL711, IL611, 
A

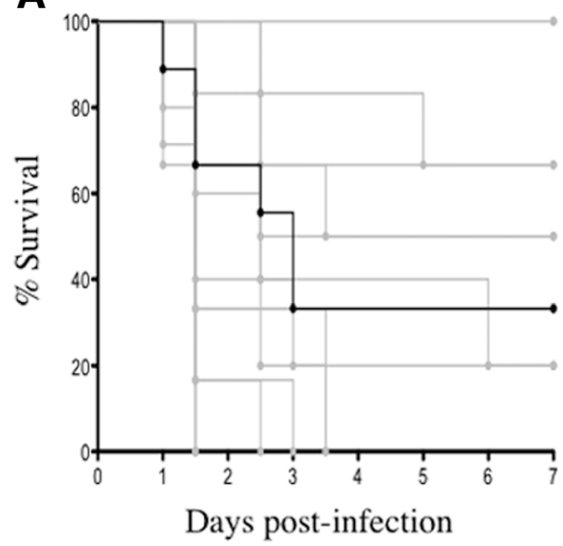

B

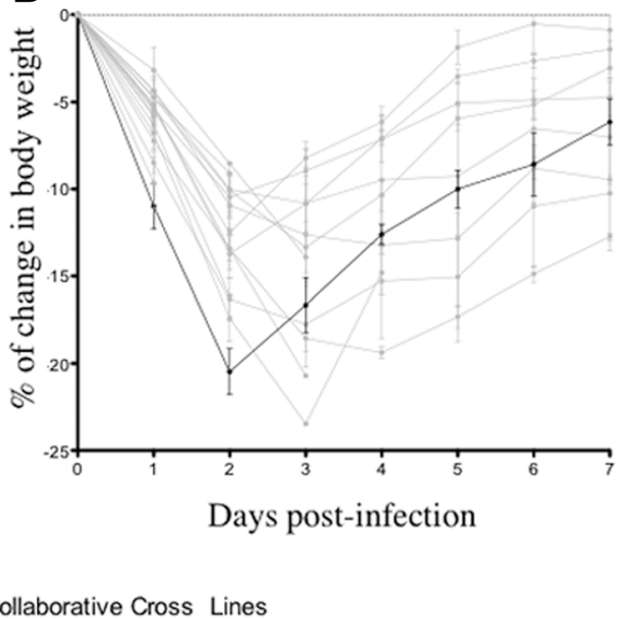

Fig 1 Disease phenotypes in CC mice during P. aeruginosa acute respiratory infection. 17 CC lines and A/J mice of 8-14 weeks old (between 3-7 mice per Line), were inoculated with a $1 \times 10^{6}$ cfu dose of the $P$. aeruginosa clinical isolate AA2, and monitored for survival time ST (a) and body weight BW (b) for a period of 7 days after infection

IL3912, IL3438, IL2156, IL2126, IL1061, IL1912, IL111; CBW1: $-4.70 \% /-6.80 \%)$ was also recorded. Overall our data show a wide range of responses to $P$. aeruginosa infection, potentially caused by allelic segregations from the eight founders strains.

\section{Effect of initial body weight, age and gender on disease phenotypes}

The effects of initial body weight or age on ST, CBW1 were tested across $17 \mathrm{CC}$ lines and A/J mice; they were found not to be significant by Spearman's Correlation (Table 1A). Similarly, gender effect on ST and CBW1 was not significantly correlated when the whole murine population was considered. Within individual CC lines no significant gender effect on disease phenotypes was observed among male and female mice (Table 1B), although a limited number of animals was consider. All together these results, emphasize the key role of genetic determinants in the severity and in the outcome of $P$. aeruginosa airway infections using this diverse murine population.

\section{Heritability of disease phenotypes during $P$. aeruginosa pneumonia}

To test if the traits variations within the infected CC murine population were due to genetic factors, we estimated heritability as described [23, 24]. Broad-sense heritability $\left(\mathrm{H}^{2}\right)$ was calculated for all the $\mathrm{CC}$ lines for which MST and CBW1 were recorded (Fig. 2c). Estimated $\mathrm{H}^{2}$ was 0.54 and 0.28 for MST and CBW1, respectively. Thus, on observation of the $\mathrm{CC}$ lines during $P$. aeruginosa infection, there is a clear host response that is affected by the genetic components as opposed to the purely environmental factors.
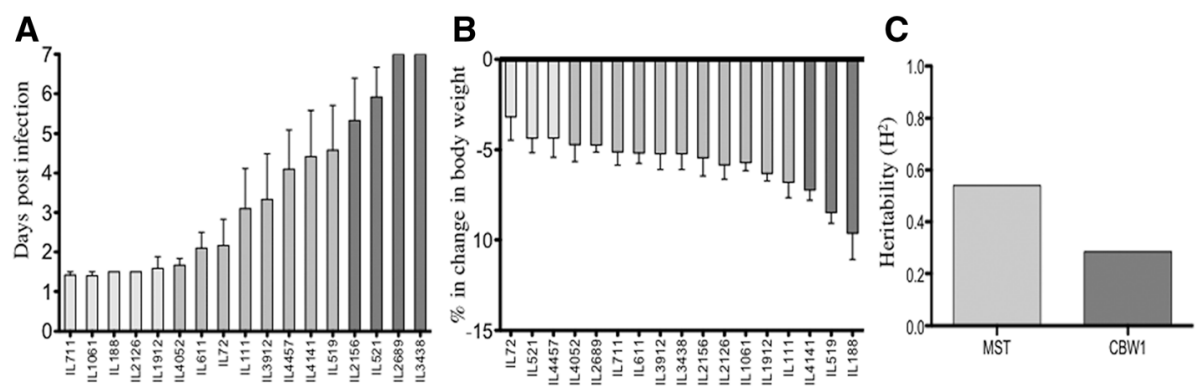

Fig 2 Evaluation of MST, CBW1 and $\mathrm{H}^{2}$ of CC lines after $P$. aeruginosa airway infection. The CC mice resource population had a strong wideresponse to $P$. aeruginosa airway infection in the MST (a) and CBW1 traits (b). MST and CBW1 of CC lines are arranged in increasing order of mean magnitude. Based on Bonferroni's Multiple Comparison Tests (BMCT) (Additional file 3: Table S3) three distinct groups have been identified among 17 CC strains infected with P. aeruginosa and are indicated as scales of grey. Estimates of broad sense $\mathrm{H}^{2}$ (c) have been evaluated for MST and CBW1, as previously described [23] 
Table 1 Analysis of initial body weight, age and gender on disease phenotypes

\begin{tabular}{|c|c|c|c|c|c|c|c|}
\hline A & \multicolumn{3}{|c|}{ Survival Time $(n=92)$} & \multicolumn{4}{|c|}{ Change in BW day $1(n=92)$} \\
\hline Initial body weight & \multicolumn{3}{|c|}{$r=0.192 \mathrm{P}>0.05$} & \multicolumn{4}{|c|}{$r=0.010 P>0.05$} \\
\hline Week's old & \multicolumn{3}{|c|}{$r=0.099 \mathrm{P}>0.05$} & \multicolumn{4}{|c|}{$r=0.173 P>0.05$} \\
\hline Gender & \multicolumn{3}{|c|}{$r=0.070 P>0.05$} & \multicolumn{4}{|c|}{$r=0.035 P>0.05$} \\
\hline \multicolumn{8}{|l|}{ B } \\
\hline \multicolumn{8}{|l|}{ Mean Survival Time } \\
\hline & \multicolumn{3}{|l|}{ Male } & \multicolumn{3}{|l|}{ Female } & ANOVA \\
\hline & Mean & SEM & $\mathrm{n}$ & Mean & SEM & $\mathrm{n}$ & $P$ value \\
\hline$A / J$ & 1.83 & 0.60 & 3 & 4.67 & 1.06 & 6 & $P>0.05$ \\
\hline IL 4052 & 1.83 & 0.33 & 3 & 1.50 & 0.00 & 3 & $P>0.05$ \\
\hline IL 3438 & 7.00 & 0.00 & 3 & 7.00 & 0.00 & 3 & $P>0.05$ \\
\hline IL 2156 & 7.00 & 0.00 & 3 & 3.67 & 1.69 & 3 & $P>0.05$ \\
\hline IL 711 & 1.50 & 0.00 & 2 & 1.30 & 0.12 & 5 & $P>0.05$ \\
\hline IL 2126 & 1.50 & 0.00 & 4 & 1.50 & 0.00 & 2 & $P>0.05$ \\
\hline IL 4141 & 4.25 & 2.75 & 2 & 4.50 & 1.46 & 4 & $P>0.05$ \\
\hline IL 4457 & 5.17 & 1.36 & 3 & 2.50 & 0.00 & 2 & $P>0.05$ \\
\hline IL 1912 & 1.17 & 0.17 & 3 & 2.00 & 0.50 & 3 & $P>0.05$ \\
\hline \multicolumn{8}{|c|}{$\%$ Change in body weight day 1} \\
\hline & \multicolumn{3}{|l|}{ Male } & \multicolumn{3}{|l|}{ Female } & ANOVA \\
\hline & Mean & SEM & $\mathrm{n}$ & Mean & SEM & $\mathrm{n}$ & $P$ value \\
\hline$A / J$ & -10.53 & 5.90 & 2 & -11.30 & 1.11 & 5 & $P>0.05$ \\
\hline IL 4052 & -3.05 & 0.94 & 3 & -6.37 & 0.91 & 3 & $P>0.05$ \\
\hline IL 3438 & -6.52 & 0.79 & 3 & -4.28 & 0.47 & 3 & $P>0.05$ \\
\hline IL 2156 & -5.69 & 1.75 & 3 & -5.18 & 1.41 & 3 & $P>0.05$ \\
\hline IL 711 & -5.13 & 1.30 & 2 & -5.10 & 1.23 & 2 & $P>0.05$ \\
\hline IL 2126 & -6.18 & 1.19 & 4 & -5.15 & 0.49 & 2 & $P>0.05$ \\
\hline IL 4141 & -8.45 & 1.27 & 2 & -6.62 & 0.43 & 4 & $P>0.05$ \\
\hline IL 4457 & -3.76 & 1.41 & 3 & -5.23 & 2.03 & 2 & $P>0.05$ \\
\hline IL 1912 & -6.36 & 0.00 & 1 & -6.29 & 0.59 & 3 & $P>0.05$ \\
\hline
\end{tabular}

Overall Spearman's correlation ( $r$ correlation coefficients and $P$ value) between all the initial parameters (initial BW, week's old and gender) of CC lines and recorded traits (ST: $n=92, C B W 1: n=92$ ) to determine potential influence (A). Two-way ANOVA's comparison across eight selected CC lines and A/J commercial inbred line to evaluate gender influence on recorded traits (MST and CWB1) (B)

\section{Discussion}

Several studies highlight how host response to infection may be strongly influenced by the cumulative effect and interactions of multiple genetic loci and by a complex set of other factors (e.g. the environment, the bacterial strain and origin, age and gender) $[1,13,25]$. Among the most relevant infectious diseases that affect humans, $P$. aeruginosa shows a wide variation in the clinical outcome in individuals at risk [8-10], indicating that the host-response may contribute to the variation of morbidity and mortality. However, predictive experimental animal models to dissect complex genetic traits, such as multiple genetic loci, that can influence the outcome of $P$. aeruginosa respiratory infection, remain to be established. To date, a poor phenotype/genotype correlation in human studies and the lack of a fully faithful mouse model have limited scientific advancements in the field [13-15]. To meet the current challenge of complex trait analysis, we used CC mice to evaluate the contribution of the host to $P$. aeruginosa pathogenicity. First, based on phenotypic diversity of human response to infection, we defined two end-points (ST and BW) that may ultimately have clinical relevance in term of morbidity and mortality. Based on previous studies with commercial inbred strains [17, 22], MST and CBW1 were selected as phenotypic disease traits to perform segregation analysis. Our approach generated wide-marked range of phenotypic differences in terms of MST and CBW1 during P. aeruginosa pneumonia, although a limited number of lines were considered. On the other, future experiments will define detailed phenotypic traits, such as bacterial load, inflammatory cytokines or cellular recruitment into the lung.

Next, initial variables - including body weight, age and gender - have limited influence on $P$. aeruginosa outcome, emphasizing the key role of genetic determinants in the disease severity in our infectious model system. In addition, the high value of the broad-sense heritability of the two recorded disease phenotypic traits support our suggestion that host genotype is an important determinant factor in the disease's severity. A similar approach was developed using Diversity Outbred mice to model Staphylococcus aureus and influenza Virus pneumonia [26]. Interestingly, they demonstrated that initial body weight correlated with viral burden, including co-infected mice, but not with $S$. aureus bacterial load. These suggest that the correlation of initial body weight and infectious graveness is modulated by the specific host-pathogen interplay. Both host genetic background and type of pathogen may have a key role in determining morbidity.

Of relevance, the power of $\mathrm{CC}$ population as model system relies also in the fact that all potential co-variables, that may influence phenotypic traits, are taken into account in the statistical analysis. Thus for future mapping of key genetic loci/genes, by using $\mathrm{CC}$ population and model of airway infection, we can track the influence of confounding effects that are bottlenecks in genetic mapping in the human population.

\section{Conclusions}

Exploring CC mouse population together with a model of respiratory infection, our results demonstrates that $P$. aeruginosa opportunistic infection has wide range of disease phenotypes affected by multiple host genetic factors, such as multiple genetic loci or polymorphic variations. Future mapping of key genetic loci/genes involved in the $P$. aeruginosa infection will be carried out with the use of additional CC lines and further traits of disease phenotypes will be assessed. This innovative approach, 
based on the concept of forward genetics, will provide new insight into the key molecular processes that control host/ pathogen interactions in respiratory disease, and should reveal novel targets for human personalized therapeutic strategies.

\section{Material and methods Ethic statement}

All experimental mice and protocols were approved by the Institutional Animal Care and Use Committee of Tel Aviv University (TAU) (approval number: M-13-079). P. aeruginosa clinical isolate AA2 was obtained from CF patient attending the Medizinische Hochschule of Hannover, Germany and described previously [17, 22, 27]. Research on the bacterial isolates from the individual with $\mathrm{CF}$ has been approved by the responsible physician at the $\mathrm{CF}$ center at Hannover Medical School, Germany. Patient gave informed consent before the sample collection. Approval for storing of biological materials was obtained by the Hannover Medical School, Germany.

\section{Collaborative cross and inbred lines}

A total of 92 (50 Males, 42 Females) mice (8 to 14-week old) from 17 different $\mathrm{CC}$ mouse lines (average 3-7 mice per line) were provided by the Small Animal Facility at Sackler Faculty of Medicine, Tel Aviv University (TAU). The lines were at inbreeding generations F20-F39, minimum $90 \%$ homozygosity. Full details of the development of these CC lines are given in Welsh et al. [3, 28]. In addition to the $\mathrm{CC}$ mice, commercially inbred lines, $\mathrm{A} / \mathrm{J}$ mice were used as internal control and were was purchased from Jackson Laboratory. The infection challenge was carried out at the BSL-2 laboratory at TAU. Mice were housed on hardwood chip bedding in open-top cages at the animal facility and were given tap water and rodent chow ad libitum.

After $P$. aeruginosa infection, mice were monitored twice per day for the parameters vocalisation, piloerection, attitude, locomotion, breathing, curiosity, nasal secretion, grooming and dehydration. Mice that lost $>25 \%$ body weight and had evidence of severe clinical disease, such as scruffy coat, inactivity, loss of appetite, poor locomotion, or painful posture, were sacrificed before the termination of the experiments with an overdose of carbon dioxide.

\section{$P$. aeruginosa strain and mouse model of acute respiratory infection}

Prior to the animal experiments, $P$. aeruginosa AA2 clinical strain was grown in Trypticase Soy Broth (TSB) to reach the exponential phase at $37{ }^{\circ} \mathrm{C}[22,27]$. The mice were anesthetized and infected by intratracheal injection with a $10^{6}$ colony forming unit (cfu) implanted into the lung via the cannula, with all lobes inoculated as described [22]. ST and BW of mice were monitored daily over one week; then the surviving mice were euthanized and tested for cfu in blood, liver and lung.

\section{Disease phenotypic traits and segregation analysis}

ST and BW of mice were considered as disease phenotypic traits for mortality and morbidity of $P$. aeruginosa pneumonia. In particular, MST and CBW1 were selected for segregation analysis, based on previous studies with commercial inbred strains $[17,22]$. At day one post $P$. aeruginosa infection, a high decrease of body weight associated to low mortality rate was reported $[17,22]$. Based on the statistical significance, using Bonferroni's Multiple Comparison Tests (reported in Additional file 3), CC lines were arranged in three groups for MTS and CBW1 by increasing order of magnitude, as described [24].

\section{Estimation of heritability}

Broad sense heritability - including epistatic but not dominance effects for MST and CBW1 - was calculated across the $C C$ lines under $P$. aeruginosa infection and in naïve conditions as described [23]. Briefly, One-way ANOVA by CC line was implemented separately for MST and CBW1. Based on these analyses, broad sense heritability $\left(\mathrm{H}^{2}\right)$, was calculated across the CC lines under control and challenge conditions, separately.

\section{Statistical analysis}

Spearman's correlation was used for associations tests. One- and Two-ANOVA and Bonferroni's Multiple Comparison Tests were used to determine the statistical significance, using GraphPad software.

\section{Additional files}

Additional file 1: Table S1. Survival Time of each CC lines after $P$. aeruginosa airway infection. (DOCX $51 \mathrm{~kb}$ )

Additional file 2: Table S2. Change in body weight of each CC line after $P$. aeruginosa airway. (DOCX $51 \mathrm{~kb}$ )

Additional file 3: Table S3. Bonferroni's Multiple Comparison Tests of recorded traits (MST and CBW1) among CC lines after $P$. aeruginosa airway infection. (DOCX $74 \mathrm{~kb}$ )

\section{Abbreviations}

CC: Collaborative Cross; ST: Survival time; MST: Mean survival time; BW: Body weight; CBW1: Change in body weight at day 1; QTL: Quantitative trait loci.

\section{Competing interests}

The authors declare no potential conflict of interest with respect to financial or Non-financial competing interests, the authorship and/or publication of this article.

\section{Authors' contributions}

NIL participated in the design of the study, carried out the mice infection, participated in data analysis and wrote the manuscript. FAl participated in data analysis and wrote the manuscript. $A B$ participated in designing the study and wrote the manuscript. All authors read and approved the final manuscript. FAI and $A B$ contributed equally to the work. NIL and $A B$ shared corresponding authorship. 


\section{Acknowledgments}

The authors thank B. Tummler (Klinische Forschergruppe, Medizinische Hochschule Hannover, Germany) for supplying the P. aeruginosa AA2 clinical strain. This study was supported to A.B. by Ministero della Salute (project GR 2009/1579812); to F.A.I. by Wellcome Trust Grant (Wellcome Trust - 085906/Z/08/ Z; 083573/Z/07/Z; 075491/Z/04); to A.B. and F.A.I. by Italian Cystic Fibrosis Research Foundation (FFC\#9/2014); to N.I.L. with a Short Term Scientific Mission (STSM) by SYSGENET network (BM0901) and European Respiratory Society Fellowship (STRTF 2014-5493).

The funders had no role in study design, data collection and analysis, decision to publish, or preparation of the manuscript.

\section{Author details}

${ }^{1}$ Infection and Cystic Fibrosis Unit, Division of Immunology, Transplantation and Infectious Diseases, IRCCS - San Raffaele Scientific Institute, Milan, Italy. 2Department of Clinical Microbiology and Immunology, Sackler Faculty of Medicine, Tel Aviv University, Ramat Aviv 69978, Tel Aviv, Israel.

\section{Received: 4 May 2015 Accepted: 3 August 2015}

Published online: 28 August 2015

\section{References}

1. Chapman SJ, Hill AV. Human genetic susceptibility to infectious disease. Nat Rev Genet. 2012;13:175-88.

2. Christaki E, Giamarellos-Bourboulis EJ. The complex pathogenesis of bacteremia: from antimicrobial clearance mechanisms to the genetic background of the host. Virulence. 2014;5:57-65.

3. Welsh CE, Miller DR, Manly KF, Wang J, McMillan L, Morahan G, et al. Status and access to the Collaborative Cross population. Mamm Genome. 2012;23:706-12.

4. Churchill GA, Airey DC, Allayee H, Angel JM, Attie AD, Beatty J, et al. The Collaborative Cross, a community resource for the genetic analysis of complex traits. Nat Genet. 2004;36:1133-7.

5. The genome architecture of the Collaborative Cross mouse genetic reference population. Genetics 2012, 190: 389-401.

6. Aylor DL, Valdar W, Foulds-Mathes W, Buus RJ, Verdugo RA, Baric RS, et al. Genetic analysis of complex traits in the emerging Collaborative Cross. Genome Res. 2011;21:1213-22.

7. Durrant C, Tayem H, Yalcin B, Cleak J, Goodstadt L, de Villena FP, et al. Collaborative Cross mice and their power to map host susceptibility to Aspergillus fumigatus infection. Genome Res. 2011;21:1239-48.

8. Boucher HW, Talbot GH, Bradley JS, Edwards JE, Gilbert D, Rice LB, et al. Bad bugs, no drugs: no ESKAPE! An update from the Infectious Diseases Society of America. Clin Infect Dis. 2009:48:1-12.

9. Gellatly SL, Hancock RE. Pseudomonas aeruginosa: new insights into pathogenesis and host defenses. Pathogens and disease. 2013;67:159-73.

10. Cigana C, Lore NI, Bernardini ML, Bragonzi A. Dampening Host Sensing and Avoiding Recognition in Pseudomonas aeruginosa Pneumonia. J Biomed Biotechnol. 2011;2011:852513.

11. Weiler CA, Drumm ML. Genetic influences on cystic fibrosis lung disease severity. Front Pharmacol. 2013;4:40.

12. Green DM, Collaco JM, MCDougal KE, Naughton KM, Blackman SM, Cutting GR. Heritability of respiratory infection with Pseudomonas aeruginosa in cystic fibrosis. J Pediatr. 2012;161:290-5. e291.

13. Schughart K, Libert C, Kas MJ. Controlling complexity: the clinical relevance of mouse complex genetics. Eur J Hum Genet. 2013;21:1191-6.

14. Ermann J, Glimcher LH. After GWAS: mice to the rescue? Curr Opin Immunol. 2012;24:564-70.

15. Psoter KJ, Rosenfeld M, De Roos AJ, Mayer JD, Wakefield J. Differential geographical risk of initial Pseudomonas aeruginosa acquisition in young US children with cystic fibrosis. Am J Epidemiol. 2014;179:1503-13.

16. Peters LL, Robledo RF, Bult CJ, Churchill GA, Paigen BJ, Svenson KL. The mouse as a model for human biology: a resource guide for complex trait analysis. Nat Rev Genet. 2007;8:58-69.

17. De Simone M, Spagnuolo L, Lore NI, Rossi G, Cigana C, De Fino I, et al. Host genetic background influences the response to the opportunistic Pseudomonas aeruginosa infection altering cell-mediated immunity and bacterial replication. PLoS One. 2014;9:e106873.

18. Rasmussen AL, Okumura A, Ferris MT, Green R, Feldmann F, Kelly SM, et al. Host genetic diversity enables Ebola hemorrhagic fever pathogenesis and resistance. Science. 2014;346:987-91.
19. Ferris MT, Aylor DL, Bottomly D, Whitmore AC, Aicher LD, Bell TA, et al. Modeling host genetic regulation of influenza pathogenesis in the collaborative cross. PLoS Pathog. 2013;9:e1003196.

20. Kollmus H, Wilk E, Schughart K. Systems biology and systems genetics-novel innovative approaches to study host-pathogen interactions during influenza infection. Current opinion in virology. 2014;6C:47-54.

21. Vered K, Durrant C, Mott R, Iraqi FA. Susceptibility to klebsiella pneumonaie infection in collaborative cross mice is a complex trait controlled by at least three loci acting at different time points. BMC Genomics. 2014;15:865.

22. Lore NI, Cigana C, De Fino I, Riva C, Juhas M, Schwager S, et al. Cystic fibrosis-niche adaptation of Pseudomonas aeruginosa reduces virulence in multiple infection hosts. PLoS One. 2012;7:e35648.

23. Iraqi FA, Athamni H, Dorman A, Salymah Y, Tomlinson I, Nashif A, et al. Heritability and coefficient of genetic variation analyses of phenotypic traits provide strong basis for high-resolution QTL mapping in the Collaborative Cross mouse genetic reference population. Mamm Genome. 2014.

24. Shusterman A, Salyma Y, Nashef A, Soller M, Wilensky A, Mott R, et al. Genotype is an important determinant factor of host susceptibility to periodontitis in the Collaborative Cross and inbred mouse populations. BMC Genet. 2013;14:68.

25. Gogos C, Kotsaki A, Pelekanou A, Giannikopoulos G, Vaki I, Maravitsa P, et al. Early alterations of the innate and adaptive immune statuses in sepsis according to the type of underlying infection. Crit Care. 2010;14:R96.

26. McHugh KJ, Mandalapu S, Kolls JK, Ross TM, Alcorn JF. A novel outbred mouse model of 2009 pandemic influenza and bacterial co-infection severity. PLoS One. 2013;8:e82865.

27. Bragonzi A, Paroni M, Nonis A, Cramer N, Montanari S, Rejman J, et al. Pseudomonas aeruginosa microevolution during cystic fibrosis lung infection establishes clones with adapted virulence. Am J Respir Crit Care Med. 2009;180:138-45.

28. Iraqi FA, Churchill G, Mott R. The Collaborative Cross, developing a resource for mammalian systems genetics: a status report of the Wellcome Trust cohort. Mamm Genome. 2008;19:379-81.

\section{Submit your next manuscript to BioMed Central and take full advantage of:}

- Convenient online submission

- Thorough peer review

- No space constraints or color figure charges

- Immediate publication on acceptance

- Inclusion in PubMed, CAS, Scopus and Google Scholar

- Research which is freely available for redistribution 